\title{
Sound Radiation From A Flow-Excited Rectangular Plate With Visco-Elastic Supports
}

\author{
Junhong Park and Luc G. Mongeau \\ School of Mechanical Engineering, 1077 Herrick Laboratories, Purdue University, West Lafayette, IN47907-1077
}

\section{Introduction}

The prediction of the sound radiated by visco-elastically supported plates excited by turbulent flows is relevant to the problem of interior vehicle noise. This subject, which has been investigated previously [1], has recently gained much interest due to the fact that aero-dynamic noise sources become more significant as other vehicle noise sources (such as the engine, the transmissions, or the tires) have been significantly reduced. In this study, a model was developed to predict the vibration response of a rectangular plate mounted on viscoelastic supports. The plate was assumed to be excited by a distributed surface pressure field, described by a Corcos model. The radiated sound was calculated from the transverse velocity of the plates. Viscoelastically supported plates can be modeled as a rectangular plate with general boundary conditions along the edges. The boundary conditions are homogeneous. The assumed mode method was used to calculate the plate response at low frequencies. The results from the assumed mode method (frequency dependent damping ratio of the plate vibration) were fed into a Statistical Energy Analysis formulation for the prediction of the velocity spectrum at high frequencies.

\section{Assumed Mode Method for Plates}

Fig. 1 shows description of the visco-elastically supported rectangular plate. From the external loads from fluctuating wall pressure, the most significant contributor to noise generation is due to flexural waves inside the plate. The equation of motion for flexural displacement (normal to the plate) in the plate is

$$
D \nabla^{4} w(x, y, t)+C \dot{w}(x, y, t)+\rho_{s} \ddot{w}(x, y, t)=-p(x, y, t)
$$

where where, D is the plate bending stiffness $\left(E h^{3} / 12\left(1-v^{2}\right)\right.$ and $\mathrm{C}$ is the damping coefficients, $\rho_{s}$ is mass per unit area of the plate. If we assume that the displacement of the plate can be described by using assumed mode analysis, the displacement of the plate is

$$
w(x, y, t)=\sum_{m, n} \Psi_{m, n}(x, y) q_{m, n}(t)
$$

where, $\Psi_{m n}(x, y, t)$ is the assumed modes of the plate and $q_{m, n}(x, y, t)$ is the generalized coordinate of the plate. For the choice of assumed modes, from the results of Allain Berry[2], Taylor expansions of displacement field are used.

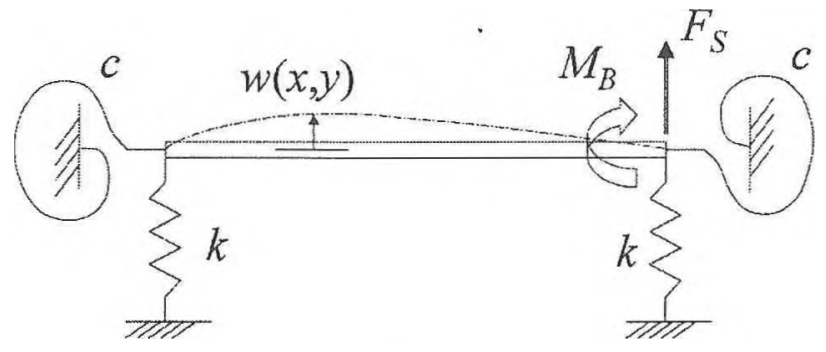

Fig. 1 description of the rectangular plate boundary conditions
The base function of assurned mode methods looks like,

$$
\phi_{m}(x)=\left(\frac{2}{a} x\right)^{m}, \quad \varphi_{n}(y)=\left(\frac{2}{b} y\right)^{n}
$$

where $a$ and $b$ are plate dimensions in $\mathrm{x}$ and $\mathrm{y}$ directions respectively. Boundaries of the plate are supported by the rotational and translational complex stiffness. Using this formulation, continuous plates problem is transformed to the $m \times n$ DOF discrete system. Mass matrix and stiffness matrix and general force matrix are calculated using the formula proposed in [2].

\section{Turbulent Wall Pressre Modeling}

The excitation from the low-Mach number turbulent flow was the topic for decades. An important contribution to the theoretical modeling of the wall pressure field was made by Corcos. Corcos postulated that the cross-power spectral density of the wall pressure can be represented as

$$
S\left(\xi_{x}, \xi_{y}, \omega\right)=\Phi_{p p}(\omega) A\left(\omega \xi_{x} / U_{c}\right) B\left(\omega \xi_{y} / U_{i}\right) \exp \left(-j \omega \xi_{x} / U_{c}\right)
$$

where $U_{c}$ is the flow convection speed, $\mathrm{A}$ and $\mathrm{B}$ are two functions that are determined from experiments. The coherence is determined from the dimensionless parameter of $\omega \xi / U_{c}$. The advantage of this Corcos model is its simplicity. For the choice of functions $A\left(\pi \xi_{x} / U_{c}\right)$ and $B\left(\varpi \xi_{y} / U_{c}\right)$, Strawderman found that, for a zero gradient flow, the functions $\mathrm{A}$ and $\mathrm{B}$ can be chosen to be simple exponentials.

$$
A\left(\omega \xi_{x} / U_{c}\right)=e^{-\gamma_{x}\left|\omega \xi_{x} / U_{c}\right|}, B\left(\omega \xi_{y} / U_{c}\right)=e^{-\gamma_{y}\left|\omega \xi_{,} / U_{c}\right|}
$$

where $\gamma_{x}$ and $\gamma_{y}$ are constants related to the ordinary coherence in the streamwise and the spanwise directions, respectively. These constants are usually determined from measurements to yield the best agreements with the experiments. The wavenumber-frequency spectral density can be obtained by performing a Fourier transform of Corcos model. The wave number-frequency spectral density is

$$
\left.\Phi\left(k_{x}, k_{y}, \omega\right)=\Phi_{p p}(\omega)\left(\frac{U_{c}}{\omega}\right)^{2} \frac{\gamma_{x}}{\pi\left[\left(k_{x} U_{c} / \omega-1\right)^{2}+\gamma_{r}\right]}\right] \frac{\gamma_{y}}{\pi\left[\left(k_{v} U_{c} /(0)^{2}+\gamma_{y}^{2}\right]\right.}
$$

This model is widely used for its mathematical simplicity. Its disadvantage is that it over predicts the sub-convective range of wave number spectrum.

\section{Experimental Apparatus}

The experiment was done using a custom-built test fixture installed in a low speed quite wind tunnel as depicted in Fig. 2. A rectangular, flat panel supported on all four edges by a glass run scaling system was mounted on a rigid aluminum frame connected to a rectangular, acoustically treated enclosure. The frame is flush with the closed test section wind tunnel floor, and structurally isolated from the vibration of the wind tunnel walls using a primary bulb sealing systems, and silicone. The wind tunnel included many vibration isolation and muffling devices. These minimized the acoustic noise in the wind tunnel test section, the turbulence level of the inflow at the 
wind tunnel section, the turbulence level of the inflow at the wind tunnel inlet, and the mechanical vibrations of the test section walls.

The test plate is made of aluminum and has the dimension of $0.446 \mathrm{~m} \times 0.375 \mathrm{~m} \times 0.003175 \mathrm{~m}$. For the simulation, the material property of aluminum was used. (Density: $2700 \mathrm{~kg} / \mathrm{m}^{3}, \mathrm{E}: 7.2 \mathrm{Gpa}, v$ : 0.34 ) The longitudinal wave speed in the plate is $5413 \mathrm{~m} / \mathrm{sec}$ and model density is 0.016542 modes $/ \mathrm{Hz}$. The aluminum plate is supported by the glass run sealing system used for actual car window. The plate itself shows very little damping ratio. When it is supported by the sealing system, damping ratio gets much higher[3]. The experimental modal analysis was used to measure the damping factor and modal frequency value. This data was used in assumed mode methods to make an analytical model of the system.

Flat fence was used to increase the turbulence level over the plate. The pressure over the plate was measured using condenser microphone (EK3024, manufactured by Emkay Products.). The wall pressure auto spectrum variation over the plate shows very little variance over the plate when the plate is placed in the region of reattached flow. The parameters for Corcos model (decay rates) was measured by comparing Corcos model to measured coherence. Convection velocity was chosen to be $0.65^{*} \mathrm{U}_{0}$ where $\mathrm{U}_{0}$ is free stream velocity. $\gamma_{x}$ and $\gamma_{y}$ was chosen as 0.37 and 0.77 respectively. Fig. 3 shows the comparison between experimental results and model in coherence variation with frequency. These results were measured at the center of the plate. Three microphone spaced $12.7 \mathrm{~mm}$ apart to each other was used for the comparison of coherence data (in stream wise direction). At the other position on the plate, it shows a little variation especially for stream wise direction.

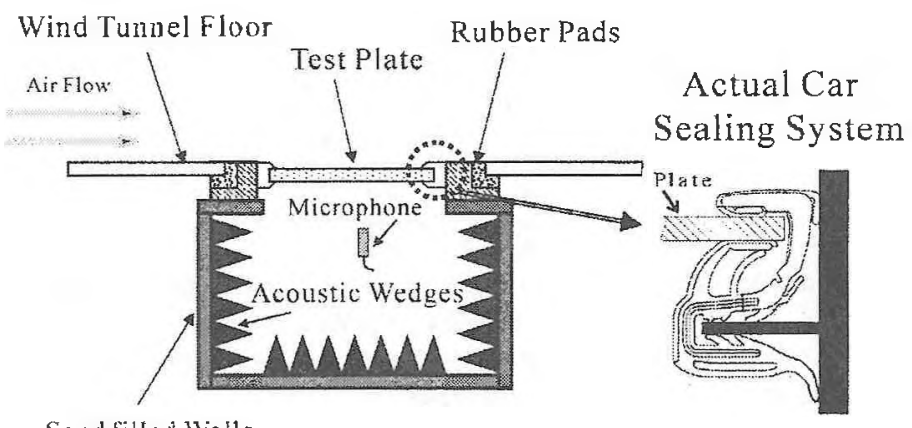

Sandfilled Walls

Fig. 2 Experimental apparatus

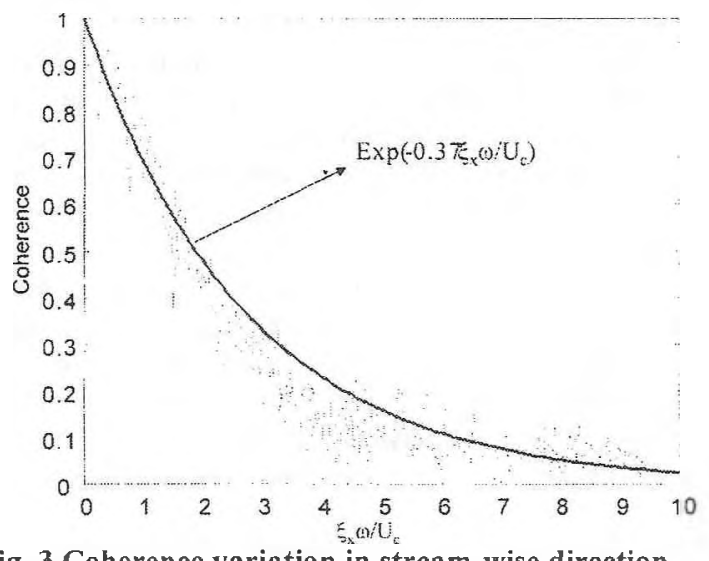

Fig. 3 Coherence variation in stream-wise direction

\section{Results and Discussions}

The response of the velocity spectrum was calculated using SEA. For the evaluation of the wave number spectrum integral of mode shape and wall pressure level, we used the assumption that all the vibration energy is constrained at the peak of mode shape wave number spectrum. [5]

The damping factor of the plate is calculated from the assumed mode method results. This damping factor is frequency dependent. For visco-elastically supported plates, the damping comes from boundary. The results from assumed mode methods shows peak value in damping ratio around $500 \mathrm{~Hz}$ and shows decay with frequency. In the SEA simulation, Corcos model over predicts the velocity spectrum significantly due to the fact that plate wave number usually corresponds to sub-convective range of the wall pressure wave number spectrum (more than $20 \mathrm{~dB}$ ). It was necessary to use refined Corcos model (Smol'yakov and Tkachenko model). Fig 4 shows the measured velocity spectrum at the center of plate and the analytical results from SEA comparison. It shows general agreement in the frequency region between $200 \mathrm{~Hz}$ and $1000 \mathrm{~Hz}$. But the error was great when it exceeds that frequency region.

\section{ACKNOWLEDGMENTS}

Thanks are expressed to Ford Motor Company for financial support and their guidance. Also, the contribution of the Herrick Lab. Technical staff is also gratefully acknowledged.

\section{REFERENCES}

[1] Han, F, Mongeau, L. G., and Bernard, R. J. 1998, "Energy flow analysis of beams and plates for random distributed loading.", Journal of Fluids and Structures, Vol. 12, pp315333.

[2] Allain Berry, Jean-Louis Guyader and Jean Nicolas. 1990, "A general formulation for the sound radiation from rectangular baffled plates with arbitrary boundary conditions." J. Acoust. Soc. Am. Vol 88 pp2792-2802.

[3] Park, J. and Mongeau, L. G., 1999, "Effects of seal mechanical properties on sound radiation from road vehicle side-glass windows." Proceedings of Inter-noise 99, pp795-800.

[4] Borisyuk, A.O. 1997, "Vibration and noise generation by elastic elements excited by a turbulent flow." Journal of Sound and Vibration, 204(2), 213-237.

[5] W. K. Blake 1986 Mechanics of Flow-induced Sound and Vibration, New York: Academic Press.

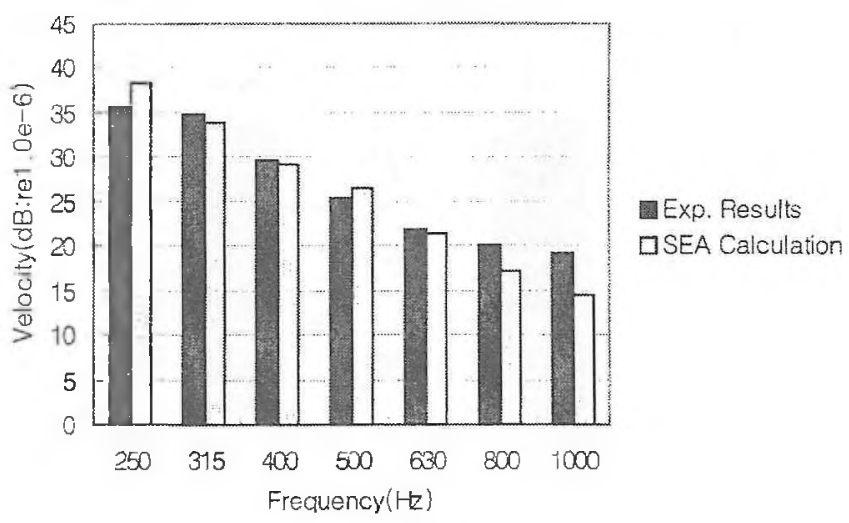

Fig. 4 Comparison between experimental result and sprediction. 